\title{
Surgery for Distant Metastatic Melanoma Improves Survival
}

\author{
Bin B. R. Kroon, MD, PhD, FRCS \\ Department of Surgery, The Netherlands Cancer Institute, Antoni van Leeuwenhoek Hospital, \\ Amsterdam, The Netherlands
}

The results of surgical treatment of stage IV melanoma patients have improved considerably over the past two decades. This is not just the result of better or less invasive operations. The tumor marker S-100B has a modest $50 \%$ positive predictive value but can draw attention to a silent distant metastasis. ${ }^{1}$ Considerable credit goes to the nuclear medicine physicians. ${ }^{18} \mathrm{~F}$-fluoro-2-deoxy-D-glucose is avidly accumulated in melanoma cells and enables positron emission tomography (PET) to discern metastases as small as $2 \mathrm{~mm}$. Hybrid PET/CT allows surgeons to pinpoint the location of a metastasis and to better plan the operation. Also, PET helps to exclude metastases elsewhere and thus selects the patients with truly limited hematogenous dissemination. As a result, the chance to cure a patient via surgery has improved, and fewer patients undergo a procedure from which they do not benefit in the end because of small metastases that become evident later on.

Howard et al. ${ }^{2}$ provide further evidence of the beneficial role of surgery for distant metastases of melanoma. Their study population consisted of 291 patients enrolled in Morton's phase III Multicenter Selective Lymphadenectomy Trial (MSLT-I) who developed distant metastases. Patients who did not receive treatment for their stage IV melanoma or whose stage IV treatment data were missing were excluded from the study. Survival was better with surgery compared to systemic treatment. The median survival of the patients who received surgery, both as single treatment or in combination with systemic medical treatment, was 15.8 months compared to 6.9 months treated with systemic medical treatment alone; 4-year survival rates were 20.8 and $7.0 \%$, respectively. The authors concluded that over half of stage IV patients are candidates for

(C) Society of Surgical Oncology 2012

Published Online: 17 May 2012

B. B. R. Kroon, MD, PhD, FRCS

e-mail: bbrkroon@gmail.com distant metastasectomy and that this approach should be undertaken whenever feasible. Strong points of the study are its size and the conscientious follow-up of the patients.

However, the study lacks the scientific soundness of a randomized design; this study thus may have had a selection bias. The authors attempt to control for this hazard by comparing surgical and nonsurgical cohorts, based on $\mathrm{M}$ stage, number of distant metastases, number of metastatic sites, and short or long disease-free interval. This approach confirmed the better survival for the surgery-containing regimen, especially for the stage M1a cohort and for the cohort of patients with one metastatic lesion. Power to confirm the survival benefit of the surgery-containing regimens can also be derived from the multivariate analysis, which revealed treatment modality and $M$ stage as significant prognostic factors.

The median survival of 11.6 months in the entire stage IV study group is about twice as long as data in the literature indicate. ${ }^{3,4}$ Careful follow-up and optimal treatment in the renowned centers taking part in the MSLT-I may explain this difference. Interesting is the finding that patients who underwent repeat surgery for recurring distant disease did better than those who received only one operation. This observation underlines once more the important role of surgery in stage IV melanoma patients.

Exciting developments in the systemic treatment of stage IV melanoma patients have been reported recently, with the $B R A F$ inhibitor vemurafinib and the monoclonal antibody ipilimumab standing out. ${ }^{5,6}$ However, the low complete response rate of these agents $(0.9$ and $1.6 \%$, respectively) and the modest increase in survival—only a few months-are not good enough to replace surgery in cases of limited distant disease. Other targeted drugs are being developed, and some are currently being tested in the clinical setting. One can foresee combinations of drugs to come out with preoperative or postoperative addition of various targeted drug-containing regimens. 
Because the major limitation the study of Howard et al. is its nonrandomized design, it is appropriate to conduct a randomized study to provide definitive proof. This is why the authors are now examining the efficacy of surgery, with or without postoperative adjuvant bacillus CalmetteGuerin immunotherapy, versus best medical therapy as initial treatment for resectable stage IV melanoma in a prospective phase III international trial. Timing of surgery versus systemic treatment is another important end point of this trial. The development of new and effective drugs-no matter how welcome these are-may prove to be a threat to the proposed study. Because different regimens are used in the various hospitals, the investigators understandably leave systemic treatment at the discretion of medical oncologists. The result may be a plethora of different regimens. If the systemic medical treatment arm turns out to be inferior, one will wonder whether an effective drug regimen was perhaps overshadowed by ineffective systemic therapies. Also, it is likely that a quick succession of additional new drugs will be administered during the study. At the end of the study, I expect the playing field to be different from what it is today, and even different from the foreseeable future. One will wonder what the relevance of the result is if the current systemic treatment comes out on top. However, these considerations should not deter the investigators from carrying on. Here is another chance for Donald Morton to lead the melanoma community to advances in care for future patients.
It has been only 3 years since a distinguished melanoma expert at the World Health Organization melanoma conference in Vienna in 2009 grumbled that "a breakthrough in systemic therapy is not on the horizon." How different the perspective is today! Now, it is realistic to predict that treatment of stage IV melanoma patients will turn multidisciplinary. The large, multicenter study by Howard et al. clearly underlines the important role of tailored surgery in such a changing treatment paradigm.

\section{REFERENCES}

1. Aukema TS, Valdés Olmos RA, Korse CM, et al. Utility of FDG PET/CT and brain MRI in melanoma patients with increased serum S-100B level during follow-up. Ann Surg Oncol. 2010;17: $1657-61$.

2. Howard JH, Thompson JF, Mozzillo N, et al. Metastasectomy for distant metastatic melanoma: analysis of data from the first Multicenter Selective Lymphadenectomy Trial (MSLT-I). Ann Surg Oncol. In press.

3. Barth A, Wanek LA, Morton DL. Prognostic factors in 1,521 melanoma patients with distant metastases. J Am Coll Surg. 1995; 181:193-201.

4. Atkins MB, Hauschild A, Wahl RL, Balch CM. Diagnosis of stage IV melanoma. In: Balch CM, Houghton AN, Sober AJ, Soong SJ, Atkins MB, Thompson JF, editors. Cutaneous melanoma. 5th ed. St. Louis: Quality Medical Publishing; 2009. p. 573-602.

5. Chapman $P B$, Hauschild $A$, Robert $C$, et al. Improved survival with vemurafinib in melanoma with BRAF V600E mutation. N Engl J Med. 2011:364:2507-16.

6. Robert C, Thomas L, Bondarenko I, et al. Ipilimumab plus dacarbazine for previously untreated metastatic melanoma. $N$ Engl J Med. 2011:364:2517-26. 